\title{
ON EELLS-SAMPSON'S EXISTENCE THEOREM FOR HARMONIC MAPS VIA EXPONENTIALLY HARMONIC MAPS
}

\author{
TOSHIAKI OMORI
}

\begin{abstract}
In this note, we introduce an approximation of harmonic maps via a sequence of exponentially harmonic maps. We then reestablish the existence theorem of harmonic maps due to Eells and Sampson.
\end{abstract}

\section{§1. Introduction}

Throughout this article, let $(M, g)$ be an $m$-dimensional compact connected Riemannian manifold without boundary, and let $(N, h)$ be an $n$ dimensional compact Riemannian manifold. A classical definition says that $u:(M, g) \rightarrow(N, h)$ is harmonic if it is a smooth critical point of the Dirichlet energy functional

$$
E(u):=\int_{M}|d u|^{2} d \mu_{g},
$$

where $|d u|$ is the Hilbert-Schmidt norm of the differential $d u$ and where $d \mu_{g}$ is the Riemannian volume element on $(M, g)$. A smooth map $u: M \rightarrow N$ is harmonic if and only if it satisfies the Euler-Lagrange equation

$$
\tau(u)=\operatorname{div}_{g}(d u)=0
$$

One of the most interesting and important subjects for harmonic maps is their existence. A typical existence problem can be formulated in the following manner:

Can a given map $f: M \rightarrow N$ be continuously deformed into a harmonic $\operatorname{map} u:(M, g) \rightarrow(N, h)$ ?

In their famous paper, Eells and Sampson [4] first concerned themselves with such a problem in the general case and proved, under the assumption that $(N, h)$ is nonpositively curved, that a given map $f: M \rightarrow N$ can

Received December 26, 2009. Accepted May 9, 2010.

2000 Mathematics Subject Classification. 53C43, 58J05.

(C) 2011 by The Editorial Board of the Nagoya Mathematical Journal 
be deformed into a harmonic map in its homotopy class. Their method is based on an analysis of the time-evolution problem corresponding to the harmonic map equation (1.1). They then proved, under the above curvature restriction, that such a time-evolution equation has a global regular solution, which converges to a harmonic map as time goes to infinity.

In this note, we consider a sequence $u_{\varepsilon}:(M, g) \rightarrow(N, h)$ of critical points of the parameterized exponential energy functional

$$
\mathbb{E}_{\varepsilon}(u):=\int_{M} e^{\varepsilon|d u|^{2}} d \mu_{g}
$$

for $\varepsilon>0$. The corresponding Euler-Lagrange equation is given by

$$
\operatorname{div}_{g}\left(e^{\varepsilon|d u|^{2}} d u\right)=e^{\varepsilon|d u|^{2}}\left\{\tau(u)+\varepsilon\left\langle\nabla|d u|^{2}, d u\right\rangle\right\}=0
$$

where $\tau(u)=\operatorname{div}_{g}(d u)$ is the tension field given in (1.1). This sequence $\left\{u_{\varepsilon}\right\}_{\varepsilon>0}$ is then expected to approximate a harmonic map as $\varepsilon \rightarrow 0$. We actually have the following.

THEOREM 1.1. Assume that the sectional curvature of $(N, h)$ is nonpositive: $\operatorname{Riem}^{N} \leq 0$. Let $\left\{u_{\varepsilon}\right\}_{\varepsilon>0}$ be a sequence of smooth critical points of the functional $\mathbb{E}_{\varepsilon}$ for $\varepsilon \rightarrow 0$ satisfying the uniform boundedness condition of energy

$$
\int_{M} \frac{e^{\varepsilon\left|d u_{\varepsilon}\right|^{2}}-1}{\varepsilon} d \mu_{g} \leq E_{0}
$$

with some constant $E_{0}>0$. Then there exists a subsequence $\left\{u_{\varepsilon(k)}\right\}_{k=1}^{\infty} \subseteq$ $\left\{u_{\varepsilon}\right\}_{\varepsilon>0}, \varepsilon(k) \rightarrow 0$ as $k \rightarrow \infty$, and a harmonic map $u:(M, g) \rightarrow(N, h)$ such that

$$
u_{\varepsilon(k)} \rightarrow u(k \rightarrow \infty) \quad \text { in } C^{\infty}(M, N)
$$

This theorem will be found to give another approach to the Eells-Sampson existence theorem in [4]. That is to say, Theorem 1.1 implies the following.

Corollary 1.2. Assume that Riem ${ }^{N} \leq 0$. Then any homotopy class of continuous maps from $M$ to $N$ admits a harmonic map.

The organization of this article is as follows. Section 2 is devoted to some preliminary issues about exponentially harmonic maps needed in the sequel. Section 3 gives complete proofs of Theorem 1.1 and Corollary 1.2. 


\section{§2. Exponentially harmonic maps}

This section provides some known results on exponentially harmonic maps, a part of which will be needed later. We start with the definition of exponentially harmonic maps, which was first introduced by Eells and Lemaire [3].

Definition 2.1. An exponentially harmonic map $u:(M, g) \rightarrow(N, h)$ is a smooth critical point of the exponential energy functional

$$
\mathbb{E}(u):=\int_{M} e^{|d u|^{2}} d \mu_{g}
$$

The Euler-Lagrange equation of this problem can be written as

$$
\operatorname{div}_{g}\left(e^{|d u|^{2}} d u\right)=e^{|d u|^{2}}\left\{\tau(u)+\left\langle\nabla|d u|^{2}, d u\right\rangle\right\}=0,
$$

where $\tau(u)=\operatorname{div}_{g}(d u)$ is the tension field of $u$.

One of the reasons why it is interesting to study the functional $\mathbb{E}$ is that the existence of its minima in a given homotopy class is always guaranteed without any special assumptions.

Proposition 2.3 (see [3]). For any homotopy class $\mathcal{H} \in[M, N]$ of continuous maps from $M$ to $N$, there exists an $\mathbb{E}$-minimizer $u$ in $\mathcal{H}$, which is necessarily $\alpha$-Hölder-continuous for any exponent $0<\alpha<1$.

This proposition follows essentially from the inequality

$$
\frac{1}{k !} \int_{M}|d u|^{2 k} d \mu_{g} \leq \int_{M} \sum_{k=0}^{\infty} \frac{1}{k !}|d u|^{2 k} d \mu_{g}=\mathbb{E}(u),
$$

which guarantees that a minimizing sequence for $\mathbb{E}$ is uniformly bounded in each Sobolev space $W^{1,2 k}(M, N)$. From the proof of this proposition in [3], however, it does not immediately follow that $u$ is smooth, or even Lipschitzcontinuous, or that it satisfies the Euler-Lagrange equation (2.2), even in a weak sense.

However, the faster the growth of a functional, the higher the regularity of its minima that we can expect. Indeed, in the case of $N=\mathbb{R}$, Duc and Eells [2] showed that an $\mathbb{E}$-minimizer $u:(M, g) \rightarrow \mathbb{R}$ of the Dirichlet problem is smooth in the interior of $M$, where $(M, g)$ is a compact Riemannian manifold with boundary, and Lieberman [7] showed the global regularity 
for $u: \Omega \rightarrow \mathbb{R}$, where $\Omega \subseteq \mathbb{R}^{m}$ is an open subset. Also, for $n \geq 2$, Naito [8] showed that an $\mathbb{E}$-minimizer $u: \Omega \rightarrow \mathbb{R}^{n}$, where $\Omega \subseteq \mathbb{R}^{m}$ is a bounded domain, is smooth in the interior of $\Omega$. Thereafter, Duc [1] at last showed the following strongest regularity theorem for $\mathbb{E}$-minimizers.

Theorem 2.4 (see [1]). Let $\mathcal{H} \in[M, N]$ be a given homotopy class. Then an $\mathbb{E}$-minimizer $u:(M, g) \rightarrow(N, h)$ in $\mathcal{H}$ is necessarily smooth.

Remark 2.5. (1) Combining this theorem with Proposition 2.3, we see that there always exists an exponentially harmonic map in a given homotopy class, which solves (2.2) in the classical sense.

(2) As mentioned in [1, Section 3], the Hölder norm $\|d u\|_{C^{\alpha}}$ of the gradient of an exponentially harmonic map $u$ is estimated by a constant depending only on $(M, g),(N, h), \mathbb{E}(u)$, and the Lipschitz constant $\|d u\|_{L^{\infty}}$. Therefore, in order to verify Theorem 1.1 , it suffices to show that $\left\|d u_{\varepsilon}\right\|_{L^{\infty}}$ is uniformly bounded as $\varepsilon \rightarrow 0$.

Also, we need the following lemmas in the proof of Theorem 1.1. Their proofs are direct calculations, so we omit them.

LemmA 2.6. If $u:(M, g) \rightarrow(N, h)$ is an exponentially harmonic map, and if we consider a homothetic transformation $h \rightarrow \varepsilon^{-1} h$, for $\varepsilon>0$, then $u:(M, g) \rightarrow\left(N, \varepsilon^{-1} h\right)$ is a critical point of the functional $\mathbb{E}_{\varepsilon}$.

Lemma 2.7. An exponentially harmonic map $u:(M, g) \rightarrow(N, h)$ satisfies the following identity of Bochner-Weitzenböck type:

$$
\begin{aligned}
S^{i j} \nabla_{i} \nabla_{j} e^{|d u|^{2}}= & 2 e^{|d u|^{2}}|\nabla d u|^{2}+2 e^{|d u|^{2}}|\tau(u)|^{2} \\
& +2 e^{|d u|^{2}} \sum_{i, j=1}^{m}\left\langle d u\left(\operatorname{Ric}^{M}\left(e_{i}, e_{j}\right) e_{j}\right), d u\left(e_{i}\right)\right\rangle \\
& -2 e^{|d u|^{2}} \sum_{i, j=1}^{m}\left\langle R^{N}\left(d u\left(e_{i}\right), d u\left(e_{j}\right)\right) d u\left(e_{j}\right), d u\left(e_{i}\right)\right\rangle,
\end{aligned}
$$

where $\operatorname{Ric}^{M}$ stands for the Ricci curvature of $(M, g), R^{N}$ stands for the curvature tensor of $(N, h),\left\{e_{i}\right\}_{i=1}^{m}$ is a local orthonormal frame field on $M$, and $S \in \Gamma(T M \otimes T M)$ is given by

$$
S^{i j}:=g^{i j}+2\left\langle d u\left(e_{i}\right), d u\left(e_{j}\right)\right\rangle \quad(i, j=1,2, \ldots, m) .
$$


We end this section by noting some basic properties similar to those of harmonic maps, which can be proved from the Bochner-Weitzenböck identity.

Corollary 2.9 (see [6]). Let $u:(M, g) \rightarrow(N, h)$ be an exponentially harmonic map. If $\operatorname{Ric}^{M} \geq 0$ and Riem $^{N} \leq 0$, then the following hold.

(1) $u$ is totally geodesic.

(2) If $\operatorname{Ric}^{M}$ is positive at some point in $M$, then $u$ is constant on $M$.

(3) If Riem $^{N}<0$ everywhere, then $u$ is either a constant or a map onto a closed geodesic in $N$.

\section{§3. Proof of the main theorem}

This section is devoted to the proof of Theorem 1.1. In what follows, by using the Nash isometric embedding $i:(N, h) \hookrightarrow \mathbb{R}^{N}$, we identify $i \circ u$ with $u$ for a map $u: M \rightarrow N$. We mean by $d u$ the derivative of $u: M \rightarrow N$, and by $\nabla u$ the gradient of the function $u: M \rightarrow N \subseteq \mathbb{R}^{N}$.

Let $B_{r} \subseteq M$ be an open ball of radius $r>0$ (centered at a fixed point of $M)$. We need the Euler-Lagrange equation of the form

$$
0=\int_{B_{r}} \nabla_{i} u^{A} \nabla^{i} \varphi^{A} e^{|\nabla u|^{2}} d \mu_{g}+\int_{B_{r}} \nabla d \Pi^{A}(u)(\nabla u, \nabla u) \varphi^{A} e^{|\nabla u|^{2}} d \mu_{g}
$$

$(A=1,2, \ldots, N)$, for any test function $\varphi \in C_{0}^{\infty}\left(B_{r}, \mathbb{R}^{N}\right)$, where $\Pi: U_{\delta}(N) \rightarrow$ $N$ is the nearest projection from a tubular neighborhood $U_{\delta}(N)$ of $N$ onto $N$. Note the relation $\nabla d i(X, Y)=\nabla d \Pi(d i(X), d i(Y))$ for $X, Y \in \Gamma(T N)$.

Our first task is to show, under the assumption that $\operatorname{Riem}^{N} \leq 0$, that the gradient of an exponentially harmonic map is bounded by a constant depending only on $(M, g)$ and its total energy and not on the target metric $h$. That is to say, we have the following.

Lemma 3.2. Assume that Riem ${ }^{N} \leq 0$. Then for any exponentially harmonic map $u:(M, g) \rightarrow(N, h)$, there exists a constant $C_{0}$ depending only on $(M, g)$, the total energy $\mathbb{E}(u)$, and not on $h$ such that

$$
\sup _{M}|\nabla u|^{2} \leq C_{0} \int_{M}\left(e^{|\nabla u|^{2}}-1\right) d \mu_{g}
$$

REMARK 3.3. Our proof of Lemma 3.2 is mainly due to the arguments in [8], which are for the case that $(M, g)$ is a Euclidean domain $\Omega$ and that $u: \Omega \rightarrow \mathbb{R}^{n}$ is an exponentially harmonic function. 
Proof of Lemma 3.2. We first consider the case of $m=\operatorname{dim} M \geq 3$. The proof has four steps.

STEP 1. There exists $\delta_{0}=\delta_{0}(m)>0$ such that

$$
\left((\sigma r)^{-m} \int_{B_{\sigma r}} e^{(1+\delta)|\nabla u|^{2}} d \mu_{g}\right)^{(m-2) / m} \leq C_{1} \frac{r^{-m}}{(1-\sigma)^{2}} \mathbb{E}(u)
$$

for all $0<\delta \leq \delta_{0}$ and $0<\sigma<1$, where $C_{1}=C_{1}(M)>0$.

As in the proof of [8, Proposition 2.10], choose $\gamma<0$ so that $\gamma>-(2 / m)$ and

$$
\varphi^{A}=\nabla^{k}\left(w^{\gamma / 2} \eta^{2} \nabla_{k} u^{A}\right)
$$

as a test function in (3.1), where $w:=e^{|\nabla u|^{2}}$ and where $\eta: B_{r} \rightarrow \mathbb{R}$ is a cutoff function satisfying

$$
0 \leq \eta \leq 1, \quad \eta=1 \text { on } B_{\sigma r}, \quad \operatorname{supp} \eta \subseteq B_{r}, \quad|\nabla \eta| \leq \frac{1}{(1-\sigma) r} .
$$

First we note that it follows from the Ricci identity that

$$
\begin{aligned}
\nabla^{i} \varphi^{A} & =\nabla^{i} \nabla^{k}\left(w^{\gamma / 2} \eta^{2} \nabla_{k} u^{A}\right) \\
& =\nabla^{k} \nabla^{i}\left(w^{\gamma / 2} \eta^{2} \nabla_{k} u^{A}\right)-g^{i j} g^{k l} R_{j l k}^{M s}\left(w^{\gamma / 2} \eta^{2} \nabla_{s} u^{A}\right),
\end{aligned}
$$

where $R^{M l}{ }_{i j k} \partial_{l}=\nabla_{\partial_{i}} \nabla_{\partial_{j}} \partial_{k}-\nabla_{\partial_{j}} \nabla_{\partial_{i}} \partial_{k}$ is the curvature tensor of $(M, g)$. Then, after the integration by parts with respect to $\nabla^{k},(3.1)$ becomes

$$
\begin{aligned}
0=\int_{B_{r}}\left(\nabla^{k} \nabla_{i} u^{A}+\nabla_{i} u^{A} \nabla^{k}|\nabla u|^{2}\right) \nabla^{i}\left(w^{\gamma / 2} \eta^{2} \nabla_{k} u^{A}\right) e^{|\nabla u|^{2}} d \mu_{g} \\
+\int_{B_{r}} \sum_{i, j=1}^{m}\left\langle d u\left(\operatorname{Ric}^{M}\left(e_{i}, e_{j}\right) e_{j}\right), d u\left(e_{i}\right)\right\rangle w^{(\gamma / 2)+1} \eta^{2} d \mu_{g} \\
\quad-\int_{B_{r}} \nabla d \Pi^{A}(u)(\nabla u, \nabla u) \nabla^{k}\left(w^{\gamma / 2} \eta^{2} \nabla_{k} u^{A}\right) e^{|\nabla u|^{2}} d \mu_{g} \\
=\int_{B_{r}}\left(\nabla^{k} \nabla_{i} u^{A}+\nabla_{i} u^{A} \nabla^{k}|\nabla u|^{2}\right) \nabla^{i} \nabla_{k} u^{A} w^{(\gamma / 2)+1} \eta^{2} d \mu_{g} \\
\quad+\frac{\gamma}{2} \int_{B_{r}}\left(\nabla^{k} \nabla_{i} u^{A}+\nabla_{i} u^{A} \nabla^{k}|\nabla u|^{2}\right) \nabla_{k} u^{A} \nabla^{i}|\nabla u|^{2} w^{(\gamma / 2)+1} \eta^{2} d \mu_{g} \\
\quad+2 \int_{B_{r}}\left(\nabla^{k} \nabla_{i} u^{A}+\nabla_{i} u^{A} \nabla^{k}|\nabla u|^{2}\right) \nabla_{k} u^{A} w^{(\gamma / 2)+1} \eta \nabla^{i} \eta d \mu_{g}
\end{aligned}
$$




$$
\begin{aligned}
& +\int_{B_{r}} \sum_{i, j=1}^{m}\left\langle d u\left(\operatorname{Ric}^{M}\left(e_{i}, e_{j}\right) e_{j}\right), d u\left(e_{i}\right)\right\rangle w^{(\gamma / 2)+1} \eta^{2} d \mu_{g} \\
& -\int_{B_{r}} \nabla d \Pi^{A}(u)(\nabla u, \nabla u) \nabla^{k}\left(w^{\gamma / 2} \eta^{2} \nabla_{k} u^{A}\right) e^{|\nabla u|^{2}} d \mu_{g}
\end{aligned}
$$

for each $A=1,2, \ldots, N$. Since $\nabla d \Pi(u)(\nabla u, \nabla u)$ is the vertical part of $\Delta u$ to $N$, the last term becomes

$$
-\int_{B_{r}}|\nabla d \Pi(u)(\nabla u, \nabla u)|^{2} w^{(\gamma / 2)+1} \eta^{2} d \mu_{g}
$$

after taking the summation with respect to $A=1,2, \ldots, N$. Also, by the Leibniz rule,

$$
\begin{aligned}
& |\nabla \nabla(i \circ u)|^{2} \\
& \quad=|\nabla d u|^{2}+g^{i k} g^{j l}\left\langle\nabla d \Pi(u)\left(\nabla_{i} u, \nabla_{j} u\right), \nabla d \Pi(u)\left(\nabla_{k} u, \nabla_{l} u\right)\right\rangle,
\end{aligned}
$$

and by the Gauss formula,

$$
\begin{aligned}
\sum_{i, j=1}^{m} & \left\langle R^{N}\left(d u\left(e_{i}\right), d u\left(e_{j}\right)\right) d u\left(e_{j}\right), d u\left(e_{i}\right)\right\rangle \\
= & |\nabla d \Pi(u)(\nabla u, \nabla u)|^{2} \\
\quad & \quad g^{i k} g^{j l}\left\langle\nabla d \Pi(u)\left(\nabla_{i} u, \nabla_{j} u\right), \nabla d \Pi(u)\left(\nabla_{k} u, \nabla_{l} u\right)\right\rangle .
\end{aligned}
$$

Substituting (3.7) and (3.8) into (3.6) after taking the summation then yields

$$
\begin{aligned}
0=\int_{B_{r}}\{ & \left.|\nabla d u|^{2}+\frac{\gamma}{2}\left|\left\langle\nabla|\nabla u|^{2}, \nabla u\right\rangle\right|^{2}\right\} w^{(\gamma / 2)+1} \eta^{2} d \mu_{g} \\
& +\left.\left.\frac{1}{2}\left(\frac{\gamma}{2}+1\right) \int_{B_{r}}|\nabla| \nabla u\right|^{2}\right|^{2} w^{(\gamma / 2)+1} \eta^{2} d \mu_{g} \\
& +\int_{B_{r}}\left\{\left\langle\nabla|\nabla u|^{2}, \nabla \eta\right\rangle\right. \\
& \left.+2 \sum_{A=1}^{N}\left\langle\nabla|\nabla u|^{2}, \nabla u^{A}\right\rangle\left\langle\nabla u^{A}, \nabla \eta\right\rangle\right\} w^{(\gamma / 2)+1} \eta d \mu_{g}
\end{aligned}
$$




$$
\begin{aligned}
& +\int_{B_{r}} \sum_{i, j=1}^{m}\left\langle d u\left(\operatorname{Ric}^{M}\left(e_{i}, e_{j}\right) e_{j}\right), d u\left(e_{i}\right)\right\rangle w^{(\gamma / 2)+1} \eta^{2} d \mu_{g} \\
& -\int_{B_{r}} \sum_{i, j=1}^{m}\left\langle R^{N}\left(d u\left(e_{i}\right), d u\left(e_{j}\right)\right) d u\left(e_{j}\right), d u\left(e_{i}\right)\right\rangle w^{(\gamma / 2)+1} \eta^{2} d \mu_{g}
\end{aligned}
$$

Here the first term is nonnegative by the choice of $\gamma>-(2 / m)$ because $u$ solves the Euler-Lagrange equation $\tau(u)+\left\langle\nabla|\nabla u|^{2}, \nabla u\right\rangle=0$. The last term is also nonnegative because $(N, h)$ is assumed to be nonpositively curved, so that

$$
\begin{aligned}
& \left.\left.\frac{1}{2}\left(\frac{\gamma}{2}+1\right) \int_{B_{r}}|\nabla| \nabla u\right|^{2}\right|^{2} w^{(\gamma / 2)+1} \eta^{2} d \mu_{g} \\
& \leq-\int_{B_{r}}\left\{\left\langle\nabla|\nabla u|^{2}, \nabla \eta\right\rangle+2 \sum_{A=1}^{N}\left\langle\nabla|\nabla u|^{2}, \nabla u^{A}\right\rangle\left\langle\nabla u^{A}, \nabla \eta\right\rangle\right\} w^{(\gamma / 2)+1} \eta d \mu_{g} \\
& \quad-\int_{B_{r}} \sum_{i, j=1}^{m}\left\langle d u\left(\operatorname{Ric}^{M}\left(e_{i}, e_{j}\right) e_{j}\right), d u\left(e_{i}\right)\right\rangle w^{(\gamma / 2)+1} \eta^{2} d \mu_{g} \\
& \leq\left. C(m) \int_{B_{r}}|\nabla| \nabla u\right|^{2}\left|\left(1+|\nabla u|^{2}\right) w^{(\gamma / 2)+1}\right| \nabla \eta \mid \eta d \mu_{g} \\
& \quad+C(M) \int_{B_{r}}|\nabla u|^{2} w^{(\gamma / 2)+1} \eta^{2} d \mu_{g} \\
& \leq\left.\frac{C(m)}{\delta} \int_{B_{r}}|\nabla| \nabla u\right|^{2}\left|w^{(\gamma / 2)+1+\delta}\right| \nabla \eta \mid \eta d \mu_{g}+\frac{C(M)}{\delta} \int_{B_{r}} w^{(\gamma / 2)+1+\delta} \eta^{2} d \mu_{g} \\
& =\frac{C(m, \gamma)}{\delta} \int_{B_{r}}\left|\nabla\left(w^{((\gamma / 2)+1) / 2}\right)\right| \eta \cdot w^{((\gamma / 2)+1) / 2+\delta}|\nabla \eta| d \mu_{g} \\
& \quad+\frac{C(M)}{\delta} \int_{B_{r}} w^{(\gamma / 2)+1+\delta} \eta^{2} d \mu_{g},
\end{aligned}
$$

where we used $x e^{x} \leq(1 / \delta) e^{(1+\delta) x}$ for all $\delta>0$ and $x \geq 0$, and $\nabla\left(|\nabla u|^{2}\right) \cdot w=$ $\nabla w$. If we choose $\delta=-(\gamma / 4)>0$, then since $((\gamma / 2)+1) / 2+\delta=1 / 2$ and $(\gamma / 2)+1+\delta<1$

$$
\begin{aligned}
(\mathrm{RHS}) \leq & \frac{4 C(m, \gamma)}{-\gamma} \int_{B_{r}}\left|\nabla\left(w^{((\gamma / 2)+1) / 2}\right)\right| \eta \cdot e^{(1 / 2)|\nabla u|^{2}}|\nabla \eta| d \mu_{g} \\
& +\frac{4 C(M)}{-\gamma} \int_{B_{r}} e^{|\nabla u|^{2}} \eta^{2} d \mu_{g} .
\end{aligned}
$$


On the other hand, the left-hand side can be written as

$$
\left.\left.\frac{1}{2}\left(\frac{\gamma}{2}+1\right) \int_{B_{r}}|\nabla| \nabla u\right|^{2}\right|^{2} w^{(\gamma / 2)+1} \eta^{2} d \mu_{g}=C(\gamma) \int_{B_{r}}\left|\nabla\left(w^{((\gamma / 2)+1) / 2}\right)\right|^{2} \eta^{2} d \mu_{g} .
$$

Therefore, after using the Young inequality, we obtain

$$
\int_{B_{r}}\left|\nabla\left(\eta w^{((\gamma / 2)+1) / 2}\right)\right|^{2} d \mu_{g} \leq \frac{C(M, \gamma)}{(1-\sigma)^{2} r^{2}} \int_{B_{r}} e^{|\nabla u|^{2}} d \mu_{g}
$$

Applying the Sobolev embedding theorem to this yields

$$
\begin{aligned}
& \left((\sigma r)^{-m} \int_{B_{\sigma r}} w^{((\gamma / 2)+1) m /(m-2)} d \mu_{g}\right)^{(m-2) / m} \\
& \quad \leq C(M, \gamma) \frac{r^{-m}}{(1-\sigma)^{2}} \int_{B_{r}} e^{|\nabla u|^{2}} d \mu_{g}
\end{aligned}
$$

which proves $(3.4)$ if we put $1+\delta_{0}:=((\gamma / 2)+1) m /(m-2)>1$ because $\gamma>-(4 / m)$.

STEP 2. There exists $1<p<m /(m-2)$ such that

$$
\begin{aligned}
& \left((\sigma r)^{-m} \int_{B_{\sigma r}} e^{\alpha m /(m-2)|\nabla u|^{2}} d \mu_{g}\right)^{(m-2) / m} \\
& \leq C_{2}\left(r^{-m} \int_{B_{r}} e^{\alpha p|\nabla u|^{2}} d \mu_{g}\right)^{1 / p}
\end{aligned}
$$

for all $\alpha \geq 1$ and $0<\sigma<1$, where

$$
C_{2}=\frac{C(M, \alpha)}{(1-\sigma)^{2}}\left(r^{-m} \int_{B_{r}} e^{\left(1+\delta_{0}\right)|\nabla u|^{2}} d \mu_{g}\right)^{1 / q}
$$

$((1 / p)+(1 / q)=1)$, and $C(M, \alpha)$ is a constant depending only on $m$ and $\left\|\operatorname{Ric}^{M}\right\|_{L^{\infty}}$ and admits at most polynomial growth in $\alpha$.

As a test function, we choose $(3.5)$ with $w=e^{|\nabla u|^{2}}$ and $\gamma \geq 0$. Then by a similar calculation,

$$
\begin{aligned}
& \int_{B_{\sigma r}}\left|\nabla\left(w^{((\gamma / 2)+1) / 2}\right)\right|^{2} d \mu_{g} \\
& \quad \leq \frac{C(M, \gamma)}{\delta} \frac{1}{(1-\sigma)^{2} r^{2}} \int_{B_{r}} w^{(\gamma / 2)+1+\delta} d \mu_{g}
\end{aligned}
$$


for any $\delta>0$, where $C(M, \gamma)$ is a constant which admits at most polynomial growth in $\gamma$. After putting $\alpha=(\gamma / 2)+1 \geq 1$, we use the Sobolev embedding theorem to obtain

$$
\begin{aligned}
& \left((\sigma r)^{-m} \int_{B_{\sigma r}} w^{\alpha m /(m-2)} d \mu_{g}\right)^{(m-2) / m} \\
& \leq \frac{C(M, \alpha)}{\delta(1-\sigma)^{2}}\left(r^{-m} \int_{B_{r}} w^{\alpha+\delta} d \mu_{g}\right) \\
& \leq \frac{C(M, \alpha)}{\delta(1-\sigma)^{2}}\left(r^{-m} \int_{B_{r}} w^{\alpha p} d \mu_{g}\right)^{1 / p}\left(r^{-m} \int_{B_{r}} w^{\delta q} d \mu_{g}\right)^{1 / q}
\end{aligned}
$$

where $(1 / p)+(1 / q)=1$. If we choose $p$ so that $1<p<m /(m-2)$ and subsequently $\delta>0$ so that $\delta q<1+\delta_{0}$, then (3.10) is obtained.

STEP 3 (Moser's iteration). There exists $C_{3}=C_{3}(M, \mathbb{E}(u))>0$ such that

$$
\sup _{M}|\nabla u| \leq C_{3}
$$

By Step 2, there exists $1<p<m /(m-2)$ such that

$$
\begin{aligned}
& \left((\sigma r)^{-m} \int_{B_{\sigma r}} w^{\alpha m /(m-2)} d \mu_{g}\right)^{(m-2) / m} \\
& \quad \leq \frac{C(M, \alpha, \mathbb{E}(u), r)}{(1-\sigma)^{2}}\left(r^{-m} \int_{B_{r}} w^{\alpha p} d \mu_{g}\right)^{1 / p}
\end{aligned}
$$

for all $\alpha \geq 1$ and $0<\sigma<1$, where $w=e^{|\nabla u|^{2}}$ and where $C(M, \alpha, \mathbb{E}(u), r)$ is a constant which admits at most polynomial growth in $\alpha$. Now we set $r_{0}:=r$, and for every $k \in \mathbb{N}$, we set

$$
r_{k}:=r \prod_{j=1}^{k} \sigma_{j}, \quad \sigma_{j}:=\frac{1+2^{-j}}{1+2^{1-j}}, \quad B_{k}:=B_{r_{k}}, \quad \alpha_{k}:=\left(\frac{1}{p} \cdot \frac{m}{m-2}\right)^{k} .
$$

Then by noting that $\alpha_{k} \geq 1$ and that $\alpha_{k} p=\alpha_{k-1} m /(m-2)$,

$$
\begin{aligned}
\left(r_{k}^{-m}\right. & \left.\int_{B_{k}} w^{\alpha_{k} m /(m-2)} d \mu_{g}\right)^{\alpha_{k}^{-1}(m-2) / m} \\
\leq & \left(\frac{C\left(M, \alpha_{k}, \mathbb{E}(u), r_{k-1}\right)}{\left(1-\sigma_{k}\right)^{2}}\right)^{\alpha_{k}^{-1}} \\
& \times\left(r_{k-1}^{-m} \int_{B_{k-1}} w^{\alpha_{k-1} m /(m-2)} d \mu_{g}\right)^{\alpha_{k-1}^{-1}(m-2) / m}
\end{aligned}
$$




$$
\begin{aligned}
\leq & \left\{\prod_{j=1}^{k}\left(\frac{C\left(M, \alpha_{j}, \mathbb{E}(u), r_{j-1}\right)}{\left(1-\sigma_{j}\right)^{2}}\right)^{\alpha_{j}^{-1}}\right\} \\
& \times\left(r^{-m} \int_{B_{r}} w^{m /(m-2)} d \mu_{g}\right)^{(m-2) / m} .
\end{aligned}
$$

Claim. The coefficient $\prod_{j=1}^{k}\left(\left(C\left(M, \alpha_{j}, \mathbb{E}(u), r_{j-1}\right)\right) /\left(1-\sigma_{j}\right)^{2}\right)^{\alpha_{j}^{-1}}$ is bounded as $k \rightarrow \infty$.

To this end, it suffices to prove that

$$
\sum_{j=1}^{k} \frac{1}{\alpha_{j}} \log \left[\frac{C\left(M, \alpha_{j}, \mathbb{E}(u), r_{j-1}\right)}{\left(1-\sigma_{j}\right)^{2}}\right]
$$

is bounded as $k \rightarrow \infty$. Since $\alpha_{j}=s^{j}, s>1$, while $C\left(M, \alpha_{j}, \mathbb{E}(u), r_{j-1}\right)$ admits at most polynomial growth in $\alpha_{j}$, it clearly follows that

$$
\sum_{j=1}^{k} \frac{1}{\alpha_{j}} \log C\left(M, \alpha_{j}, \mathbb{E}(u), r_{j-1}\right)
$$

is bounded as $k \rightarrow \infty$. Furthermore, by the choice of $\sigma_{j}$, we see that

$$
\sum_{j=1}^{k} \frac{1}{\alpha_{j}} \log \frac{1}{\left(1-\sigma_{j}\right)^{2}}=\sum_{j=1}^{k} \frac{1}{s^{j}} \log \frac{\left(1+2^{-j}\right)^{2}}{2^{-2 j}} \leq \sum_{j=1}^{k} \frac{1}{s^{j}} \log \left(4^{j+1}\right),
$$

which is clearly bounded as $k \rightarrow \infty$. This proves the claim.

Hence, we can take the limit $k \rightarrow \infty$ in (3.12) to obtain

$$
\begin{aligned}
\sup _{B_{r / 2}}|\nabla u|^{2} & \leq \sup _{B_{r / 2}} w \\
& \leq C\left(r^{-m} \int_{B_{r}} e^{m /(m-2)|\nabla u|^{2}} d \mu_{g}\right)^{(m-2) / m} \\
& \leq C_{3}(M, \mathbb{E}(u))
\end{aligned}
$$

proving (3.11).

STEP 4. There exists a constant $C_{0}=C_{0}(M, \mathbb{E}(u))>0$ such that

$$
\sup _{M}|\nabla u|^{2} \leq C_{0} \int_{M}\left(e^{|\nabla u|^{2}}-1\right) d \mu_{g}
$$


which proves Lemma 3.2.

Lemma 2.7 and (3.11), combined with the curvature assumption that Riem $^{N} \leq 0$, imply that

$$
\begin{aligned}
& S^{i j} \nabla_{i} \nabla_{j}\left(e^{|\nabla u|^{2}}-1\right)=S^{i j} \nabla_{i} \nabla_{j} e^{|\nabla u|^{2}} \\
& \geq 2 e^{|\nabla u|^{2}} \sum_{i, j=1}^{m}\left\langle d u\left(\operatorname{Ric}^{M}\left(e_{i}, e_{j}\right) e_{j}\right), d u\left(e_{i}\right)\right\rangle \\
& \geq-C\left(m,\left\|\operatorname{Ric}^{M}\right\|_{L^{\infty}}\right) e^{|\nabla u|^{2}}|\nabla u|^{2} \\
& \geq-C\left(m,\left\|\operatorname{Ric}^{M}\right\|_{L^{\infty}}, e^{\|\nabla u\|_{L}^{2}}\right)\left(e^{|\nabla u|^{2}}-1\right) .
\end{aligned}
$$

In the fourth line, we have used the inequality $|\nabla u|^{2} \leq e^{|\nabla u|^{2}}-1$. Moreover, (3.11) then guarantees that the leading term $S^{i j}$ of (2.8),

$$
S^{i j}=g^{i j}+2 h_{\alpha \beta} \nabla_{e_{i}} u^{\alpha} \nabla_{e_{j}} u^{\beta},
$$

has the bounded eigenvalues both from above and from below by a constant depending only on $(M, g)$ and $\mathbb{E}(u)$. This observation enables us to successfully apply the maximum principle (see [5, Theorem 9.20]) to acquire

$$
|\nabla u|^{2} \leq e^{|\nabla u|^{2}}-1 \leq C_{0}(M, \mathbb{E}(u)) \int_{M}\left(e^{|\nabla u|^{2}}-1\right) d \mu_{g}
$$

This proves (3.13), and we now complete the proof of Lemma 3.2 in the case of $m \geq 3$.

The proof in the case of $m=2$ is a slight modification of the above arguments.

STEP 1. Fix $1<q_{0}<2$. Then there exists $\delta_{0}=\delta_{0}\left(q_{0}\right)>0$ such that

$$
\left((\sigma r)^{-2} \int_{B_{\sigma r}} e^{(1+\delta)|\nabla u|^{2}} d \mu_{g}\right)^{1 / q_{0}} \leq C_{1} \frac{r^{-2}}{(1-\sigma)^{2}} \mathbb{E}(u)
$$

for all $0<\delta \leq \delta_{0}$ and $0<\sigma<1$, where $C_{1}=C_{1}(M)$.

To this end, taking $0>\gamma>2\left(1 / q_{0}-1\right)$ and applying the Sobolev embedding theorem to $(3.9)$, we obtain

$$
\left((\sigma r)^{-2} \int_{B_{\sigma r}} e^{((\gamma / 2)+1) q_{0}|\nabla u|^{2}} d \mu_{g}\right)^{1 / q_{0}} \leq C(M, \gamma) \frac{r^{-2}}{(1-\sigma)^{2}} \int_{B_{r}} e^{|\nabla u|^{2}} d \mu_{g}
$$

which proves $(3.14)$ if we put $1+\delta_{0}=((\gamma / 2)+1) q_{0}>1$. 
STEP 2. There exists $1<p<q_{0}$ such that

$$
\left((\sigma r)^{-2} \int_{B_{\sigma r}} e^{\alpha q_{0}|\nabla u|^{2}} d \mu_{g}\right)^{1 / q_{0}} \leq C_{2}\left(r^{-2} \int_{B_{r}} e^{\alpha p|\nabla u|^{2}} d \mu_{g}\right)^{1 / p}
$$

for all $\alpha \geq 1$ and $0<\sigma<1$, where

$$
C_{2}=\frac{C(M, \alpha)}{(1-\sigma)^{2}}\left(r^{-2} \int_{B_{r}} e^{\left(1+\delta_{0}\right)|\nabla u|^{2}} d \mu_{g}\right)^{1 / q}
$$

$((1 / p)+(1 / q)=1)$, and $C(M, \alpha)$ is a constant depending only on $(M, g)$ and admits at most polynomial growth in $\alpha$.

By using (3.15), we can apply Moser's iteration to obtain the bound (3.11) of the gradient of $u$, and the same argument as in Step 4 above is also valid in this case, which proves Lemma 3.2 in the case of $m=2$.

Proof of Theorem 1.1. Let $u_{\varepsilon}:(M, g) \rightarrow(N, h)$ be a sequence of critical points of the functional $\mathbb{E}_{\varepsilon}$ as $\varepsilon \rightarrow 0$ satisfying

$$
\int_{M} \frac{e^{\varepsilon\left|\nabla u_{\varepsilon}\right|^{2}}-1}{\varepsilon} d \mu_{g} \leq E_{0}
$$

As is mentioned in Remark 2.5(2), to complete the proof, it is enough to show that $\left\|\nabla u_{\varepsilon}\right\|_{L^{\infty}}$ is uniformly bounded as $\varepsilon \rightarrow 0$.

If we consider the homothetic transformation $h \rightarrow h_{\varepsilon}:=\varepsilon h$, then by Lemma 2.6, each $u_{\varepsilon}:(M, g) \rightarrow\left(N, h_{\varepsilon}\right)$ is an exponentially harmonic map. Then as a consequence of Lemma 3.2, we have

$$
\begin{aligned}
\varepsilon\left|\nabla u_{\varepsilon}\right|_{h}^{2}=\left|\nabla u_{\varepsilon}\right|_{h_{\varepsilon}}^{2} & \leq C\left(M, E_{0}\right) \int_{M}\left(e^{\left|\nabla u_{\varepsilon}\right|_{h_{\varepsilon}}^{2}}-1\right) d \mu_{g} \\
& =C\left(M, E_{0}\right) \int_{M}\left(e^{\varepsilon\left|\nabla u_{\varepsilon}\right|_{h}^{2}}-1\right) d \mu_{g}
\end{aligned}
$$

(Note that (3.16) implies that the total energy $\mathbb{E}\left(u_{\varepsilon}\right)$ with respect to $h_{\varepsilon}$, which is equal to $\mathbb{E}_{\varepsilon}\left(u_{\varepsilon}\right)$ with respect to $h$, is bounded by $E_{0}$. Also, note that the curvature assumption that Riem $^{N} \leq 0$ does not change under the homothetic transformation.)

Dividing (3.17) by $\varepsilon$ yields

$$
\left|\nabla u_{\varepsilon}\right|_{h}^{2} \leq C\left(M, E_{0}\right) \int_{M} \frac{e^{\varepsilon\left|\nabla u_{\varepsilon}\right|_{h}^{2}}-1}{\varepsilon} d \mu_{g} \leq C\left(M, E_{0}\right) E_{0}
$$

for all $\varepsilon>0$, which proves Theorem 1.1. 
Proof of Corollary 1.2. Let $\varphi \in \mathcal{H}$ be any smooth map. Theorem 2.4 then implies that there exists, for each $\varepsilon>0$, a smooth map $u_{\varepsilon}:(M, g) \rightarrow(N, h)$ which minimizes $\mathbb{E}_{\varepsilon}$ in $\mathcal{H}$. Since the resulting sequence $\left\{u_{\varepsilon}\right\}_{\varepsilon>0}$ satisfies

$$
\int_{M} \frac{e^{\varepsilon\left|d u_{\varepsilon}\right|^{2}}-1}{\varepsilon} d \mu_{g} \leq \int_{M} \frac{e^{\varepsilon|d \varphi|^{2}}-1}{\varepsilon} d \mu_{g}
$$

taking the limit as $\varepsilon \rightarrow 0$ yields

$$
\limsup _{\varepsilon \rightarrow 0} \int_{M} \frac{e^{\varepsilon\left|d u_{\varepsilon}\right|^{2}}-1}{\varepsilon} d \mu_{g} \leq \limsup _{\varepsilon \rightarrow 0} \int_{M} \frac{e^{\varepsilon|d \varphi|^{2}}-1}{\varepsilon} d \mu_{g}=\int_{M}|d \varphi|^{2} d \mu_{g} .
$$

This implies that some subsequence of $\left\{u_{\varepsilon}\right\}_{\varepsilon>0}$ satisfies the uniform boundedness condition of energy in Theorem 1.1, so that it moreover contains a subsequence which converges uniformly to some harmonic map $u:(M, g) \rightarrow$ $(N, h)$. The obtained harmonic map $u$ represents the homotopy class $\mathcal{H}$.

Acknowledgment. The author thanks Professor Shigetoshi Bando for suggesting this research and for his constant encouragement during this work.

\section{REFERENCES}

[1] D. M. Duc, Variational problems of certain functionals, Internat. J. Math. 6 (1995), 503-535.

[2] D. M. Duc and J. Eells, Regularity of exponentially harmonic functions, Internat. J. Math. 2 (1991), 395-408.

[3] J. Eells and L. Lemaire, "Some properties of exponentially harmonic maps" in Partial Differential Equations, Part 1, 2 (Warsaw, 1990), Banach Center Publ. 27, Part 1, Vol. 2, Polish Acad. Sci., Warsaw, 1992, 129-136.

[4] J. Eells and J. H. Sampson, Harmonic mappings of Riemannian manifolds, Amer. J. Math. 86 (1964), 109-160.

[5] D. Gilbarg and N. S. Trudinger, Elliptic partial differential equations of second order, reprint of the 1998 original, Classics Math., Springer, Berlin, 2001.

[6] J. Q. Hong and Y. H. Yang, Some results on exponentially harmonic maps (in Chinese), Chinese Ann. Math. Ser. A 14 (1993), 686-691.

[7] G. M. Lieberman, On the regularity of the minimizer of a functional with exponential growth, Comment. Math. Univ. Carolin. 33 (1992), 45-49.

[8] H. Naito, On a local Hölder continuity for a minimizer of the exponential energy functional, Nagoya Math. J. 129 (1993), 97-113.

Mathematical Institute

Tohoku University

Sendai 980-8578

Japan

sa6m07@math.tohoku.ac.jp 